\title{
Alkaloids from Piper sarmentosum and Piper nigrum
}

\begin{abstract}
Detailed chemical studies on the roots of Piper sarmentosum and Piper nigrum have resulted in several alkaloids. The roots of $\mathrm{P}$. sarmentosum gave a new aromatic compound, 1nitrosoimino-2,4,5-trimethoxybenzene (1). Piper nigrum roots gave pellitorine (2), (E)-1[3',4' (methylenedioxy)cinnamoyl]piperidine (3), 2,4-tetradecadienoic acid isobutyl amide (4), piperine (5), sylvamide (6), cepharadione A (7), piperolactam D (8) and paprazine (9). Structural elucidation of these compounds was achieved through NMR and MS techniques. Cytotoxic activity screening of the plant extracts indicated some activity.
\end{abstract}

Keyword: Piper nigrum; Piper sarmentosum; Alkaloids; Cytotoxic 